\title{
Discentes com Necessidades Especiais e os Desafios no Ensino de Algoritmos com Atividades Colaborativa e Competitiva: as regras do jogo na sala de aula
}

\author{
Alex S. Vieira ${ }^{1}$, Léia S. Sousa ${ }^{1}$ Jonh R. Belarmino, ${ }^{1}$ and Erick S. Sales ${ }^{1}$ \\ ${ }^{1}$ FACEEL/IGE - Universidade Federal do Sul e Sudeste do Pará (UNIFESSPA)
}

\begin{abstract}
Teaching and learning process of Programming is a challenge because it presents several variables such as the teacher's dynamics, the students' learning style, and programming language. Gamification or collaboration can be used to smooth the impact of these challenges. However, they want new adaptations when there are students in the class with special needs. This paper presents an experiment in a computer class, including students with special needs, in collaborative and competitive activities. The results indicate that collaborative activities are more effective, but should be planned to include students with disabilities fairly.
\end{abstract}

Resumo. O processo de ensino e aprendizagem de Programação é desafiador, pois apresenta diversas variáveis como, por exemplo, a dinâmica do professor, o estilo de aprendizagem dos alunos e a apropriação de uma linguagem de programação. Abordagens envolvendo aspectos de gamificação podem suavizar o impacto desses desafios. No entanto, elas requerem novas adaptações quando, na turma, há discentes com necessidades especiais. Este trabalho apresenta um relato de experiência em uma turma da Computação, incluindo alunos com necessidades especiais, em atividades individuais, colaborativas e competitivas. Os resultados indicam que atividades colaborativas são mais efetivas, porém devem ser planejadas para incluir, com equidade, os discentes com deficiência.

\section{Introdução}

Há diversas metodologias ativas que colocam o discente no centro do processo de ensino e aprendizagem, tornando-o cada vez mais engajado nesse processo, todavia não são triviais as abordagens que direcionam a aplicação dessas metodologias quando, no centro, estão discentes com necessidades [Stiegler and Zimmermann 2015][Silva et al. 2018]. Por outro lado, a utilização de uma metodologia ativa, justifica-se por se tratar de uma abordagem relativamente nova, mas ao mesmo tempo bastante utilizada em pesquisas da Computação, com as quais os ganhos na motivação dos alunos [Chaves et al. 2015] e retenção do conhecimento são algumas das principais vantagens.

Disciplinas como Programação podem ser desafiadoras porque dependem de variáveis como a dificuldade do professor medir com objetividade a aprendizagem do aluno, a pluralidade de estilos de aprendizagem [Ferreira et al. 2017], aprender a sintaxe de uma linguagem de programação (LP) [de Araújo et al. 2013], além de exigir habilidades tais como o raciocínio lógico, noções de inglês e diferentes formas de representações de soluções - como fluxogramas e pseudocódigos. Os desafios de ensinar Programação podem ser agravados, no ensino superior, quando há o ingresso de discentes com necessidades especiais, pois outros fatores adicionais como o despreparo ou falta de formação 
VIII Congresso Brasileiro de Informática na Educação (CBIE 2019)

Anais dos Workshops do VIII Congresso Brasileiro de Informática na Educação (WCBIE 2019)

dos docentes para receber esses discentes [Sarmento et al. 2017] dificultam o processo de inclusão.

No entanto, há diversos experimentos com aspectos de gamificação ou jogos [Borges et al. 2013] [Machado et al. 2011] [Silva et al. 2018] [De Paula and Fávero 2016] [Whittinghill 2014], atividades colaborativas[Serdyukov 2017] como aprendizagem baseada em projetos [Swart 2015] e aprendizagem baseada em problemas [Sarmento et al. 2017], que tendem a tornar o processo de ensino e aprendizagem na área de Computação mais efetivo, natural ou envolvente. Uma questão observada em relação às metodologias ativas é a dificuldade de encontrar orientações direcionadas à atividades que promovam a remoção de "qualquer [...] obstáculo que limite [...] o livre acesso" à movimentação de pessoas com deficiência [Barbosa and Silva 2010], pois essas metodologias muitas vezes negligenciam questões relativas à acessibilidade [Francisco et al. 2017][Stiegler and Zimmermann 2015].

O objetivo principal deste trabalho apresentar um relato de experiência sobre gamificação, no qual são implementadas atividades individuais, colaborativas e competitivas, em uma turma de calouros da área da Computação, que tem discentes com necessidades especiais, especificamente paralisia cerebral e baixa visão. Também é um objetivo deste trabalho, verificar qual dessas atividades é mais efetiva para esses discentes.

O experimento ocorreu no primeiro bimestre da disciplina de uma disciplina introdutória de Programação, de uma universidade federal da região norte. A turma foi dividida em 4 equipes com 6 alunos cada uma. As atividades foram implementadas no formato de um torneio, consistindo de 3 rodadas progressivas. Apenas avançava para a rodada seguinte, as equipes vencedoras da rodada anterior.

Informa-se que o uso de qualquer métrica de pontuação relacionada ao tempo foi descartada deste processo por uma questão de respeito às limitações dos alunos com necessidades especiais, visando adaptar o torneio para uma abordagem mais inclusiva.

Esse trabalho está estruturado da seguinte forma. A Seção 2 apresenta a fundamentação teórica . A Seção 3 apresenta a metodologia utilizada. A Seção 4 apresenta um experimento sobre a realização de um torneio em sala. A Seção 5 apresenta os resultados parciais e discussões. Por fim, a Seção 6 apresenta as considerações finais.

\section{Fundamentação Teórica}

Aprendizagem com gameficação em conformidade com aspectos de acessibilidade constitui um dos grandes desafios destacados na literatura sobre educação. A respeito disso, [Silva et al. 2018] foca de acessibilidade para surdos, enquanto [Stiegler and Zimmermann 2015] destaca ainda questões relacionadas à outros tipos de deficiência, como deficiência na fala e de aprendizagem, entre outros. Assim, em [Silva et al. 2018] é apresentada uma revisão sistemática da literatura sobre acessibilidade em ambientes de cursos massivos online (MOOCs) para surdos. Por outro lado, [Stiegler and Zimmermann 2015] explora questões de acesso à tecnologia digital e gamificação para considerando as necessidades dos alunos que se identificam como deficientes, e levanta importantes investigações sobre equidade e acesso a materiais tecnológicos de instrução, principalmente para deficiências cognitiva e neurológica. Já em [Whittinghill 2014], por exemplo, embora o trabalho não seja centrado no processo educacional, gamificação é utilizada como um estímulo no processo de fisioterapia dos braços 
VIII Congresso Brasileiro de Informática na Educação (CBIE 2019)

Anais dos Workshops do VIII Congresso Brasileiro de Informática na Educação (WCBIE 2019)

em pessoas com paralisia cerebral. Os autores constataram a maior satisfação e motivação dos pacientes na realização das atividades. O campo de estudos das metodologias de ensino com acessibilidade requerem muitas pesquisas ainda, em especial no que se refere ao ensino de programação para alunos com paralisia cerebral.

A própria abordagem da gamificação no ensino levanta muitas dúvidas quanto à sua implementação, como é mostrado em [De Paula and Fávero 2016]. Neste trabalho é apresentado um levantamento sobre a compreensão dos profissionais da educação a cerca de gamificação da educação, no qual é demonstrada a existência de discrepâncias entre os entendimentos e percepções sobre gamificação. Ainda assim, os autores concluíram que jogos e a gamificação são bem aceitos como ferramentas a serem empregadas em diferentes modelos pedagógicos. Entretanto, aspectos sobre acessibilidade não são citados. Por outro lado, o estudo dos estilos de aprendizagem encontra-se mais difundido na literatura. Como mostrado em [Ferreira et al. 2017], os autores implementam algoritmos de inteligência artificial no ambiente de aprendizagem Moodle para realizar um levantamento dos estilos de aprendizagem dos alunos de um curso que utilizavam essa plataforma.

Este trabalho baseou-se nos estilos de aprendizagem dos alunos (levantados no início das aulas) para definir uma proposta com o uso de elementos da gamificação. Diferente de [Silva et al. 2018] e [Stiegler and Zimmermann 2015], que se concentram na análise da literatura e levantamento de informações, este trabalho apresenta um relato de experiência com alunos com necessidades especiais, mais especificamente, paralisia cerebral e baixa visão. A abordagem de gamificação seguida é baseada em nos conceitos de desafios, engajamento, socialização e aprimoramento de habilidades sintetizados em [Borges et al. 2013], e diferente de [De Paula and Fávero 2016], que realiza um levantamento sobre a utilização de gamificação com jogos digitais, este trabalho foca em jogos não-digitais. O motivo para o uso desta estratégia é a possibilidade de promover cooperação assistida entre alunos com deficiência e sem deficiência, uma vez que este trabalho se deu no período inicial das aulas da disciplina de Programação em uma turma de alunos calouros, o que requereu observações sobre como os alunos se integrariam na resolução de problemas. Outro motivo importante é que o uso de jogos provê feedbakcs relativamente imediatos e constantes, que inclui informações sobre precisão (por exemplo, se uma questão está certa ou errada) e fornece orientação de como está ocorrendo o processo de aprendizagem do conteúdo.

\section{Metodologia}

Nesta seção apresentam-se as principais etapas de realização deste trabalho, abrangendo aspectos de colaboração e competitividade, fragmento do conteúdo programático de um Projeto Pedagógico do Curso (PPC) - da área de Computação - perfil dos estudantes e ferramentas utilizadas.

A primeira etapa (Etapa 1) corresponde às aulas tradicionais, em que o professor organiza e distribui o material didático. Nesta etapa são apresentados, em sala, problemas que aprofundam os assuntos desse PPC, os quais são resolvidos junto com os alunos. Depois são selecionados os assuntos iniciais do conteúdo programático que serão abordados no torneio.

É importante informar que, em sala de aula, o discente com paralisia cerebral é continuamente assistido por dois bolsistas, de outros cursos, em atividades como, por 
VIII Congresso Brasileiro de Informática na Educação (CBIE 2019)

Anais dos Workshops do VIII Congresso Brasileiro de Informática na Educação (WCBIE 2019)

exemplo, copiar anotações feita na lousa e intermediar a comunicação com o docente, pois a fala desse aluno é um pouco comprometida devido à sua condição. No entanto, sendo assistido pelos bolsistas, o aluno consegue participar de todas as atividades realizadas em sala.

Na segunda etapa (Etapa 2), cada discente elabora um conjunto de 6 problemas envolvendo assuntos que são apresentados adiante na Tabela 2, da Seção 4. Em seguida, esses problemas são transformados em pseudocódigos. Essas tarefas são realizadas pelos pelos discentes no período extra sala e seguem um modelo padrão - apresentado na Seção 3.1 - disponibilizado pelo docente.

A Etapa 2 deve ser realizada fora da sala, pois os discentes dispõem de mais tempo para realizar as atividades após as aulas. Isso é essencial para alunos com necessidades especiais que possuem, por exemplo, paralisia cerebral ou baixa visão, consigam concluir as tarefas no seu próprio ritmo.

$\mathrm{Na}$ terceira etapa (Etapa 3), os discentes trazem os problemas e pseudocódigos elaborados por eles, na Etapa 2, e se organizam em grupo. Depois deliberam sobre a correta formulação de suas questões e selecionam, em consenso, 3 pseudocódigos para representar os cartões-desafio do grupo. Essa etapa é importante porque possibilita que os discentes, com ou sem necessidades especiais, opinem e interajam sobre os cartões selecionados, além de proporcionar uma reflexão colaborativa e inclusiva.

A quarta etapa (Etapa 4) consiste da realização do torneio, em sala de aula. Nesta etapa, por meio do método de observação [Barbosa and Silva 2010], o professor verifica o comportamento das equipes, durante as partidas, primando pela interação e evolução das repostas de cada grupo. Ao final, tem-se a aplicação de 1 questionário para os alunos, contendo perguntas sobre o grau de motivação, satisfação e aprendizagem, utilizando a escala [Schleich et al. 2006], mas de 3 pontos para representar os qualificadores de satisfação ou motivação.

Por fim, empregou-se o modelo de torneio - muito utilizado em práticas desportivas - que consiste em partidas disputadas entre equipes adversárias, mediante a avaliação da maior pontuação. Esse modelo não necessariamente requer associação com o tempo cronometrado - o que é útil quando há pessoas com necessidades especiais participando. Neste trabalho, os pontos não são cumulativos, isto é, a cada nova rodada a contagem dos pontos é reiniciada.

\subsection{Ferramentas}

Foi utilizada a plataforma webcup para gerenciar o placar das disputas entre os grupos; o Google Classroom, utilizado para compartilhar material didático como slides, apostilas e listas de exercícios; e, para coletar a percepção da turma sobre a implantação dessas abordagens, foi utilizado o Google Formulário, com perguntas fechadas.

Adicionalmente, informa-se que foi criado um template próprio de cartão-desafio para padronizar a apresentação dos pseudocódigos dos alunos. Cada cartão consiste em um problema e um pseudocódigo contendo 5 erros implantados intencionalmente. Ele possui 3 áreas principais: área para inserção do comando da questão; área de declaração do pseudocódigo; e área contendo um teste de mesa com exemplo de entrada e saída de dados esperados. 
VIII Congresso Brasileiro de Informática na Educação (CBIE 2019)

Anais dos Workshops do VIII Congresso Brasileiro de Informática na Educação (WCBIE 2019)

\subsection{Perfil dos estudantes}

Os discentes da turma são calouros do curso da área de Computação, com média de idade entre 19 e 25 anos. Somam-se 30 discentes regularmente matriculados numa disciplina de Programação que está sendo re-ofertada. Dentre eles, um possui paralisia cerebral e outro possui baixa visão - ambos distribuídos em grupos distintos. Trata-se de um conjunto de discentes, que reprovaram em Programação, no semestre anterior.

\section{O Torneio: um Experimento em uma Turma com Discentes com Necessidades Especiais}

O torneio foi realizado com base nos pseudocódigos elaborados pelos discentes. Os pseudocódigos de uma equipe eram verificados pelas equipes adversárias. Cada equipe elegeu um representante para escrever o pseudocódigo na lousa - conforme pode ser visto na Figura 1(a). Assim, todos podiam acompanhar o desenvolvimento de cada partida. A Figura 1(b) representa o momento em que as equipes conferem os bugs. Os alunos da turma se dividiram em 4 equipes de até 6 alunos cada uma, sem restrição de critério de escolha.

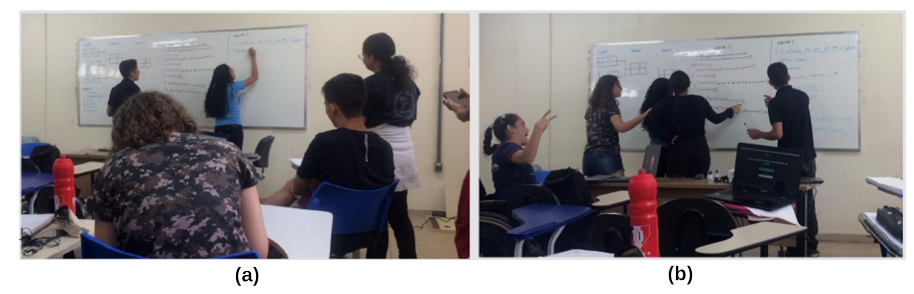

Figura 1. Apresentação dos cartões-desafio e conferência de bugs

\subsection{As Regras do Jogo}

O torneio foi projetado para realizar três rodadas e seguir um conjunto de regras próprias, as quais são apresentadas na Tabela 1. Para concorrer em cada uma dessas rodadas, a equipe precisava de um cartão-desafio com assuntos relacionados a cada rodada. A Tabela 2 apresenta a síntese desses assuntos.

Na primeira rodada (Tabela 2, coluna 2) os cartões-desafio abordam assuntos operadores e ordem de precedência. Na segunda rodada, os cartões abordam os tópicos de estrutura de controle de fluxo (condicionais ou repetição). Já na terceira rodada, rodada final, o cartão-desafio de cada equipe é composto pelos diferentes assuntos vistos na Etapa 1 e Etapa 2. Os vencedores em cada rodada seguem para a rodada seguinte. As rodadas possuem pontuações progressivas (Tabela 2, coluna 4) que são atribuídas às equipes conforme elas avançam no torneio.

\section{Resultados Parciais e Discussões}

Nesta seção são apresentados os resultados parciais observados durante a realização do torneio, com a participação de 1 discente com paralisia cerebral e 1 discente com baixa visão, em uma turma de 30 alunos regularmente matriculados na disciplina, dos quais 24 participaram do torneio realizado, no entanto, 18 deles responderam ao questionário de satisfação e motivação. 
VIII Congresso Brasileiro de Informática na Educação (CBIE 2019)

Anais dos Workshops do VIII Congresso Brasileiro de Informática na Educação (WCBIE 2019)

Tabela 1. As Regras do Jogo

\begin{tabular}{|c|c|c|}
\hline Regras & Penalidades & Exemplo de bug \\
\hline $\begin{array}{l}\text { O grupo seleciona um cartão-desafio } \\
\text { referente aos tópicos do respectivo nível. } \\
\text { O professor realiza sorteio dos pares } \\
\text { de grupos que disputarão as partidas da primeira } \\
\text { rodada. } \\
\text { Em cada disputa, dois grupos trocam seus } \\
\text { cartões-desafio e, em seguida, verificam } \\
\text { os bugs contidos. } \\
\text { Cada bug vale um ponto. } \\
\text { Devem ser inseridos } 5 \text { bugs no pseudocódigo. } \\
\text { Cada grupo deve encontrar o maior número de } \\
\text { bugs no pseudocódigo do adversário para } \\
\text { avançar para a rodada seguinte. } \\
\text { Em caso de empate, um novo cartão-desafio } \\
\text { deve ser usado como fator de desempate. } \\
\text { Na terceira rodada, o grupo que encontrar o } \\
\text { maior número de erros vence o torneio. }\end{array}$ & $\begin{array}{l}\text { Inserir mais do que } 5 \text { bugs } \\
\text { decrementa } 2 \text { pontos } \\
\text { do grupo que o projetou. } \\
\text { Inserir menos do que } 5 \text { bugs } \\
\text { decrementa } 2 \text { pontos } \\
\text { do grupo o projetou. } \\
\text { Comando de questão } \\
\text { que seja ambígua, } \\
\text { inconclusiva ou } \\
\text { incompleta não anula } \\
\text { o bug encontrado pelo } \\
\text { grupo adversário. }\end{array}$ & $\begin{array}{l}\text { - Não identar pseudocódigo. } \\
\text { - Declarar nomes de variáveis } \\
\text { com caracteres especiais. } \\
\text { - Não declarar variável } \\
\text { utilizada no processamento. } \\
\text { - Declarar variáveis de entrada } \\
\text { com nomes escritos de } \\
\text { formas diferentes. } \\
\text { - Utilizar ponto e vírgula como } \\
\text { separador de variáveis no } \\
\text { lugar da vírgula. } \\
\text { - Definir um tipo de dados } \\
\text { numérico para um problema } \\
\text { que trata de tipo literal. }\end{array}$ \\
\hline
\end{tabular}

Tabela 2. Rodadas do Torneio e Assuntos relacionados a cada rodada

\begin{tabular}{|l|l|c|c|}
\hline Rodadas & \multicolumn{1}{|c|}{ Assuntos Relacionados } & Acerto & Pontuação \\
\hline Primeira & $\begin{array}{l}\text { Operadores (atribuição, aritméticos, } \\
\text { relacionais, lógicos) e ordem de precedência }\end{array}$ & $\begin{array}{c}\text { Avanço para a } \\
\text { rodada seguinte }\end{array}$ & 0.5 \\
\hline Segunda & $\begin{array}{l}\text { Estrutura de controle de fluxo } \\
\text { (condicionais ou repetição) }\end{array}$ & $\begin{array}{c}\text { Avanço para a } \\
\text { rodada seguinte }\end{array}$ & 0.75 \\
\hline Terceira & Assuntos da Primeira e Segunda rodada & - & 1 \\
\hline
\end{tabular}

Em relação à satisfação e motivação, os alunos responderam questões quanto ao grau de aprendizagem e quanto o grau de motivação, sob 3 grupos de modalidades distintas: atividade individual, com ênfase na resolução de listas de exercícios; atividade colaborativa, com ênfase na discussão em grupo para o aperfeiçoamento do código; e atividade competitiva, com ênfase na localização de bugs.

Os resultados são apresentados respectivamente na Figura 2 e Figura 3, nas quais o eixo vertical apresenta, de baixo para cima, as modalidades atividade individual, atividade colaborativa e atividade competitiva, enquanto que o eixo horizontal representa o número de discentes que responderam o questionário.

Essas modalidades foram classificadas por 3 rótulos: irrelevante (faixa mais à esquerda), possui o mais baixo nível de avaliação; pouco relevante (faixa central), possui um grau moderado de relevância; e muito relevante (faixa mais à direita), possui um grau elevado de relevância.

Quanto à retenção de aprendizagem, na atividade individual, da Figura 2, observase que 3 discentes informaram ser irrelevante aprender sozinho, enquanto que 6 informaram que há um pouco de ganho de aprendizagem quando se estuda sozinho os algoritmos representados em pseudocódigos, e 9 discentes informaram que é muito relevante estudar isso sozinho. Define-se, neste trabalho, que estudar sozinho o pseudocódigo é o mesmo que responder a lista de exercícios sozinho.

Na atividade colaborativa, da Figura 2, observa-se que 1 discente informou ser 
VIII Congresso Brasileiro de Informática na Educação (CBIE 2019)

Anais dos Workshops do VIII Congresso Brasileiro de Informática na Educação (WCBIE 2019)

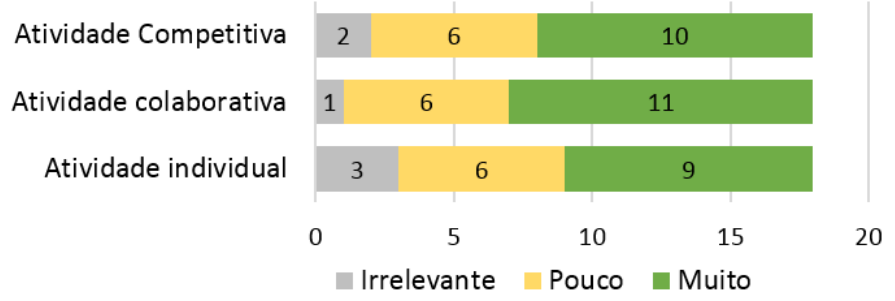

Figura 2. Resultado parcial referente à retenção de aprendizagem

irrelevante estudar com colegas, ou seja, ele considera que aprende pouco nessa modalidade, enquanto que 6 discentes informaram que é um pouco relevante estudar em grupo, e 11 discentes informaram que é muito relevante estudar esse conteúdo com a colaboração de outros colegas.

Na atividade competitiva, 2 discentes informaram que é irrelevante estudar o pseudocódigo de outros colegas, ou seja, entendem que eles não conseguem aprender analisando o pseudocódigo de terceiros. No entanto 6 alunos disseram que é um pouco relevante estudar esses pseudocódigos, e 10 discentes informaram que é muito relevante estudar o pseudocódigo de terceiros, ou seja, eles disseram que aprendem mais com essa abordagem.

Com base no questionário de satisfação quanto à retenção de aprendizagem, é possível observar que os alunos, da turma avaliada, apresentam melhores resultados quando realizam a atividade colaborativa. Em seguida, os resultados referentes à atividade competitiva podem representar um sensível aumento na retenção da aprendizagem deles, em relação a atividade individual. A ratificação da percepção de aprendizagem será verificada por meio de outros instrumentos em um próximo trabalho.

Quanto à motivação dos discentes, na atividade individual, da Figura 3, observase que 5 discentes informaram que não é relevante a motivação quando se estuda sozinho, ou seja, não se sentem motivados a estudarem sozinhos os algoritmos. Mas 8 estudantes informaram que é um pouco relevante a motivação enquanto se estuda sozinho, isto é, se sentem um pouco motivados ao estuda-los, enquanto que 5 discentes informaram que é muito relevante, para eles, estudar pseudocódigos sozinhos.

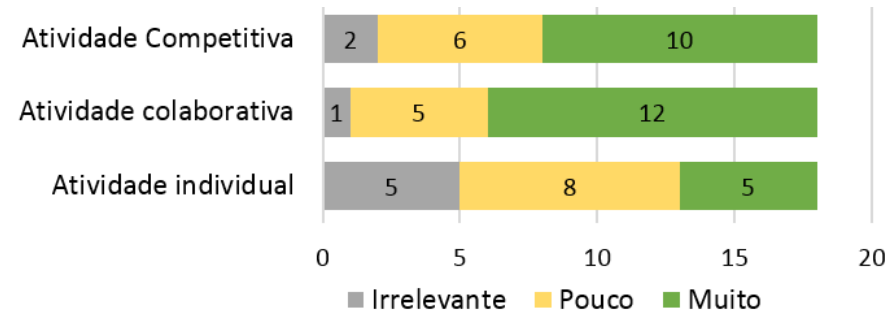

Figura 3. Resultado parcial referente à motivação

$\mathrm{Na}$ atividade colaborativa, da Figura 3, é possível observar que 1 discente informou não sentir-se motivado a trabalhar de maneira colaborativa, enquanto que 5 discentes informaram que se sentem um pouco motivados quando estudam pseudocódigos com outros colegas. E 12 discentes informaram que se setem muito motivados quando estudam, em grupo, os assuntos do torneio. 
VIII Congresso Brasileiro de Informática na Educação (CBIE 2019)

Anais dos Workshops do VIII Congresso Brasileiro de Informática na Educação (WCBIE 2019)

Na atividade competitiva, da Figura 3, há 2 discentes que informaram não se sentir motivados a estudarem algoritmos analisando os pseudocódigos de outros colegas; 6 discentes informaram que é um pouco relevante analisar o pseudocódigo de outros grupos, e 10 disseram que se sentem muito motivados quando estão analisando o pseudocódigo dos outros colegas.

A partir dos resultados obtidos no questionário de motivação, é possível dizer que os discentes se sentem mais motivados a estudar (algoritmos e sua representação em pseudocódigo) quando realizam atividades colaborativas. A segunda abordagem preferida, por essa turma, são as atividades competitivas, apesar da diferença entre essas duas não ser muito contrastante, ambas são mais empolgantes para esses alunos do que as atividades individuais.

Em relação à eficácia do torneio, em sala, com a participação de discentes com necessidades especiais é possível verificar que, de modo geral, os alunos conseguiram cumprir a atividade individual - elaboração de pseudocódigos no tempo fora de aula. E, em sala, eles conseguiram se organizar em grupos de até 6 pessoas, conforme foi estabelecido previamente.

No entanto, não foi possível concluir a primeira rodada, mesmo sendo 3 aulas de 45 minutos dedicadas para essa atividade. Uma possível explicação para isso foi o investimento de tempo considerável para debater as questões relacionadas à seleção dos 3 pseudocódigos, e também no aperfeiçoamento deles.

Além disso, durante a execução das atividades de correção dos pseudocódigos, alguns grupos empataram. O empate evidenciou um problema observado nesse tipo de abordagem que é o uso habitual do tempo como critério de avaliação. Uma possível solução seria aplicar um novo problema para os grupos empatados tentarem resolver e, declarar como vencedor, o primeiro que encontrasse todas os bugs.

No entanto, enfatiza-se que, ao trabalhar com discentes com deficiência física ou mobilidade reduzida, qualquer solução que esteja associada ao tempo, em sala de aula, como fator competitivo, torna-se um meio injusto de avaliar, devido às limitações desses alunos, pois eles conseguem resolver as questões, mas necessitam de mais tempo que a maioria dos alunos devido à sua condição.

O desempate recorrente não foi previsto, neste trabalho, para ser desassociado ao tempo. E a realização do torneio sob essa métrica acabaria impedindo a participação dos discentes com necessidades especiais de participarem efetivamente do processo, por isso o processo de seleção de um novo pseudocódigo e verificação de bugs não resolve esse problema.

Observa-se que, durante o processo de encontrar os erros implantados no pseudocódigo do grupo oponente, notou-se uma intensa interação entre os discentes que estavam resolvendo os problemas e os demais discentes que também participaram indagando sobre questões nos pseudocódigos escritos na lousa.

Por fim, encontrar um critério de desempate desassociado de critérios relacionado é um problema recorrente que precisa ser revisitado, com a finalidade de encontrar uma solução viável e inclusiva para todos. É recorrente porque podem haver mais de um empate consecutivo. 
VIII Congresso Brasileiro de Informática na Educação (CBIE 2019)

Anais dos Workshops do VIII Congresso Brasileiro de Informática na Educação (WCBIE 2019)

\section{Considerações Finais}

Este trabalho utilizou-se de elementos de gamificação, por meio de um torneio nãodigital, para ensinar os conceitos básicos de uma disciplina introdutória de Linguagem de Programação (LP) em uma turma de calouros, da área de Computação, que possui um aluno com paralisia cerebral e um aluno com baixa visão. Nesse experimento, foram inseridas atividades individuais, colaborativas e competitivas ao longo do torneio.

A turma de egressos, em questão, é composta por discentes que reprovaram no semestre anterior em LP, no qual as aulas eram divididas entre teoria e prática (por meio de exercícios). No entanto, por meio do método de observação, foi possível comparar a abordagem tradicional de ensino com a inserção de torneio, que se mostrou uma forma estimulante para a turma, na qual foi possível notar a aceitação positiva das atividades apresentadas por meio do maior engajamento da turma no desenvolvimento dessas atividades.

Mais especificamente, conforme o questionário aplicado, notou-se que a maioria da turma prefere aprender algoritmos de forma colaborativa e competitiva (constatado pelos resultados apresentados na Seção 5), a estudar esses conceitos sem o auxílio de seus colegas. A maioria deles também se sentem menos motivados quando estudam algoritmos (e suas representações) sozinhos, mas se sentem mais motivados quando fazem isso de maneira colaborativa e competitiva.

Alguns problemas foram constatados no processo de realização do experimento como, por exemplo, não poder utilizar o tempo como critério de desempate das equipes, a falta de experiência quanto ao domínio de tempo nas atividades em sala - tendo em vista a participação de alunos com necessidades especiais - e a localização de poucos trabalhos detalhando o uso de metodologias ativas e acessibilidade no ensino superior.

Como trabalhos futuros pretende-se executar novos torneios, utilizando os códigos dos alunos, para verificar se há algum desvio padrão na aprendizagem e motivação deles que esteja relacionado ao conteúdo programático. Futuramente, também devem ser projetadas e implantadas novas métricas, incluindo uma investigação qualitativa, de desempates que sejam inclusivas e aderentes às diferentes metodologias ativas.

\section{Referências}

Barbosa, S. and Silva, B. (2010). Interação humano-computador. Elsevier Brasil.

Borges, S. d. S., Reis, H. M., Durelli, V. H., Bittencourt, I. I., Jaques, P. A., and Isotani, S. (2013). Gamificação aplicada à educação: um mapeamento sistemático. In Brazilian Symposium on Computers in Education (Simpósio Brasileiro de Informática na Educação-SBIE), volume 24, page 234.

Chaves, R. O. et al. (2015). Um jogo sério para apoio ao ensino de modelagem de processo de software baseado em mapas conceituais: uma avaliação experimental.

de Araújo, A. L. S. O., Scaico, P. D., de Paiva, L. F., de Morais Rabêlo, H., de Luna Santos, L., Pessoa, F. I. R., Targino, J. M., and dos Santos Costa, L. (2013). Aplicação da taxonomia de bloom no ensino de programação com scratch. In Anais do Workshop de Informática na Escola, volume 1, page 31. 
VIII Congresso Brasileiro de Informática na Educação (CBIE 2019)

Anais dos Workshops do VIII Congresso Brasileiro de Informática na Educação (WCBIE 2019)

De Paula, F. R. and Fávero, R. (2016). A gamificação da educação na compreensão dos profissionais da educação. Proceedings of SBGames 2016.

Ferreira, L., Gazzola, M., Ferrari, D., Zupelari, M., Paiva, P., and Rodrigues Jr, J. (2017). Métodos de classificação aplicados à detecção automática de estilos de aprendizagem em um ambiente real de ensino. In Brazilian Symposium on Computers in Education (Simpósio Brasileiro de Informática na Educação-SBIE), volume 28, page 1517.

Francisco, T. H. A., Vefago, Y. B., Ramos, A. M., and de Souza, I. R. (2017). Para além das metodologias ativas: uma reflexão sobre a indução da qualidade em cursos de administração. Revista Brasileira de Ensino Superior, 3(2):38-57.

Machado, L. d. S., Moraes, R. M. d., Nunes, F. d. L. d. S., and Costa, R. M. E. M. d. (2011). Serious games baseados em realidade virtual para educação médica. Rev. bras. educ. méd, 35(2):254-262.

Sarmento, A., Farias, M., and Santos, H. (2017). Relato de experiência do pibid: Promovendo o ensino de computação de forma interdisciplinar com português no ensino fundamental. In Anais do Workshop de Informática na Escola, volume 23, page 313.

Schleich, A. L. R., Polydoro, S. A. J., and dos Santos, A. A. A. (2006). Escala de satisfação com a experiência acadêmica de estudantes do ensino superior. Avaliação psicológica, 5(1):11-20.

Serdyukov, P. (2017). Innovation in education: what works, what doesn't, and what to do about it? Journal of Research in Innovative Teaching \& Learning, 10(1):4-33.

Silva, A., Ramos, B., Biagiotti, B., Fadel, L., and Ulbricht, V. (2018). Gamificação e acessibilidade na educação de surdos: desenvolvimento de um protótipo a partir do MOOC TeleLab, pages 131-149.

Stiegler, A. and Zimmermann, G. (2015). Gamification and accessibility. In International Conference on Human Aspects of IT for the Aged Population, pages 145-154. Springer.

Swart, A. J. (2015). Distance learning engineering students languish under project-based learning, but thrive in case studies and practical workshops. IEEE Transactions on Education, 59(2):98-104.

Whittinghill, D. M. (2014). Gamification of physical therapy for the treatment of pediatric cerebral palsy: a pilot study examining player preferences. age, 24:1. 